\title{
América Latina a inicios de la segunda década del siglo XXI: entre el regionalismo estratégico y la regionalización fragmentada
}

Latin America in the beginning of the second decade of the 21st century: between the strategic regionalism and the fragmented regionalization

LINCOLN BIZZOZERO*

Rev. Bras. Polít. Int. 54 (1): 29-43 [2011]

\section{Introducción}

Durante la primera década del siglo XXI se produjo un cambio de orientación en varios gobiernos latinoamericanos, cuya manifestación común se formuló a través de una posición contraria al «modelo neoliberal». Este cambio de orientación de los gobiernos nacionales se expresó en agendas regionales con contenidos y prioridades diferentes a las que lo antecedieron en los años noventa. Las diferencias en prioridades y contenidos se han manifestado de distintas maneras: en el rechazo de algunas iniciativas (Acuerdo de Libre Comercio de las Américas); en nuevas propuestas sudamericanas (Comunidad Sudamericana de Naciones y UNASUR, el Banco del Sur y el Consejo Sudamericano de Defensa); en instancias orientadas a la cooperación entre los pueblos (ALBA); la apuesta a la generación de nuevos espacios de concertación (América Latina-Caribe).

Estas manifestaciones podrían dar a entender que se está asistiendo a un regionalismo diferente al de los años noventa del siglo pasado. Sin embargo, para llegar a esta afirmación, es necesario remitirnos a algunos parámetros que posibiliten la comparación entre uno y otro regionalismo, ya sea en los objetivos, en los principios de funcionamiento, en los instrumentos para llevar a cabo los objetivos, o en las realizaciones, lo cual implica referirse a la estrategia del proceso. Desde la perspectiva de las relaciones internacionales importa visualizar el significado del regionalismo para el Estado, si en definitiva le otorga una mayor presencia y autonomía en el sistema (desde una periferia receptora) o bien le posibilita llevar adelante una propuesta ordenadora de conjunto. Desde la perspectiva de la economía política, el regionalismo es una respuesta que pretende el desarrollo

* Profesor de la Universidad Nacional de la República, Uruguay (lbizzozero@gmail.com). 
regional y humano y para ello resulta clave el papel del Estado en la vinculación con el mercado y la sociedad. Estas dos perspectivas serán las utilizadas para evaluar el devenir del regionalismo latinoamericano: la correspondencia con las prioridades de política exterior de los Estados y el potencial que le agrega al Estado-región en su posición en el sistema el participar en un espacio regional; y el «modelo» que adopta el Estado tanto en «ideas» como en instrumentos y opciones para aplicar la política en la vinculación con la sociedad y el mercado.

En este artículo se trata de dar respuestas a los interrogantes que surgen en la díada cambio/continuidad del regionalismo latinoamericano, a si nos encontramos con un regionalismo «estratégico» y el sentido y contenidos del mismo en el contexto internacional del siglo XXI. También se analizan y plantean los fundamentos por los cuales el regionalismo resulta funcional y constituye una de las respuestas necesarias para la gobernanza mundial en el sistema internacional presente. Finalmente se intenta responder a las consecuencias que genera el regionalismo en un sistema internacional en transición: si los bloques regionales tenderán a conformar polos para constituir una parte de un sistema internacional que tendrá polos de poder (y negociación) en algunos Estados, de acuerdo a los diversos temas o si solamente cumplirá una función de adaptación (y ajuste) a los requerimientos de paz y seguridad y otras funciones de articulación con las instancias internacionales.

El trabajo continuará con una sección donde se analizará la evolución del regionalismo latinoamericano en la década de los noventa y las respuestas desde el Estado en el marco regional frente al cambio del sistema internacional y las iniciativas de Estados Unidos.

En otro apartado se tratará específicamente la vinculación funcional del Estado y del regionalismo con la evolución del capitalismo, lo cual posibilita una comprensión sobre los sustentos ideológicos que apoyan el ingreso de las potencias emergentes (PEMS) y las regiones en el diseño de un orden internacional diferente al que se perfilara en la inmediata posguerra fría de los años noventa.

En la siguiente sección se visualizará el diseño de este nuevo orden y las perspectivas del espacio latinoamericano/caribeño y de las diversas manifestaciones regionales en el mismo. Finalmente, se presentarán algunas conclusiones sobre la evolución del regionalismo en América Latina, el papel de las PEMS y de las regiones en el sistema internacional y sobre las opciones estratégicas que se le presentan a los Estados del espacio latinoamericano/caribeño en la participación en la estructura del orden internacional y en el diseño de la forma y contenidos de la gobernanza mundial.

\section{El azaroso camino latinoamericano de los noventa: entre el nuevo panamericanismo y la respuesta regional}

A fines de los años ochenta se produjo el final de un determinado sistema internacional, que durante varias décadas se manifestó a partir de una estructura 
bipolar. En esos años, América Latina estuvo confrontada a una agenda de temas que tenían como prioridades la democracia, la canalización de la deuda externa, la reforma del Estado y de la economía y la apertura comercial.

La década de los noventa comenzó con dos cambios mayores que afectaron a las bases del sistema internacional y que patentaron el pasaje a una estructura diferente, cuyo ordenamiento se basó en los recursos de poder de Estados Unidos: el final de la bipolaridad y la aceleración del proceso de mundialización del capitalismo, como consecuencia, entre otras cosas, del derrumbe del bloque socialista. La aceleración del proceso de mundialización del capitalismo se manifestó en el funcionamiento mundial de los actores económicos desde los pilares productivos de la economía mundial, es decir en los mismos lugares de gestación de la riqueza, y fue acompañado por un impulso a la regionalización desde tres polos: Estados Unidos, Europa y Japón.

Las bases de esta conformación del sistema internacional con una estructura cuyo funcionamiento ha estado garantizado por Estados Unidos, pero que ha sido acompañado en algunas funciones por otras regiones-Estados, planteó diversas interpretaciones que dejaron sin resolver varios temas atinentes al funcionamiento del sistema. Entre esos temas, una cuestión que no es menor, tiene relación con el Estado y su autopercepción, sea burocrática, de la sociedad o de ambas a la vez, sobre el papel que puede cumplir en el mundo.

América Latina no participó del impulso a la regionalización en función de una mejor inserción competitiva y tampoco de la conformación de la nueva agenda internacional, tanto en los temas políticos como económicos. La agenda latinoamericana quedó fijada temporalmente en el «ajuste» y las reformas a un «modelo» (de funcionamiento de la economía y del Estado), cuando de lo que se trataba era de modificar las pautas de funcionamiento de un sistema. De esta manera, América Latina, tanto por su posición en la periferia del sistema internacional como por su efectiva participación en los recursos de poder mundial y por la propia propuesta cultural de participación en el sistema, se quedó con una agenda donde los temas de la política mundial y de la economía política quedaron circunscriptos a un único camino para una mejor inserción competitiva.

La cuestión es que, en ese diseño primerizo del nuevo orden internacional, se consideraba que la regionalización consistía en la respuesta adecuada a las decisiones que atañían a los temas de la agenda internacional (básicamente de ordenamiento de la economía). Sin embargo, la regionalización en cuanto respuesta, al no considerar el papel de los Estados, dejaba un vacío en la articulación de las decisiones, sobre todo en temas sensibles atinentes a las sociedades nacionales.

La respuesta de los países en América Latina se originó a partir de las iniciativas de Estados Unidos, cuyo denominador común fueron las reformas a implementar del «Consenso de Washington». La Iniciativa de las Américas que propulsó el presidente de los Estados Unidos en 1990 buscó agrupar las distintas realidades regionales latinoamericanas. 
Las distintas respuestas de los países latinoamericanos elaboradas e implementadas a través de las políticas exteriores estuvieron pautadas por diversos factores, entre los cuales contaron: la cercanía e importancia del país en relación a Estados Unidos, el nivel de desarrollo institucional y humano, las capacidades en recursos de poder y finalmente la definición política sobre el papel a cumplir en el escenario internacional. La "fotografía» que se visualizó a inicios de los noventa fue un collage, como resultado de la iniciativa de Estados Unidos de "panamericanismo abierto" y las respuestas desde los países de América Latina. Las respuestas fueron desde la aceptación de la propuesta panamericana, a un incipiente llamado al «regionalismo sudamericano», pasando por un difuso y renovado espacio latinoamericano y la opción de «separarse» de los países de la región, para priorizar o integrarse al mercado más importante del mundo.

La materialización de las prioridades estadounidenses derivó en la inclusión de México para la conformación del Tratado de Libre Comercio de América del Norte (TLCAN) y el inicio de negociaciones con Chile para un TLC durante la década de los noventa. El MERCOSUR surgió en el contexto del Consenso de Washington impulsado por gobiernos liberales y posibilitó algunos cambios necesarios para una inserción competitiva, pero también constituyó una herramienta para la proyección de las prioridades internacionales de los países, en particular de Argentina y Brasil. En ese sentido, la conformación del MERCOSUR generó una doble respuesta: de inserción competitiva y también estratégica, desde el momento que para los socios grandes, y en particular para Brasil, el MERCOSUR se definió como política exterior del Estado frente al proceso de mundialización.

El considerar al MERCOSUR como «regionalismo estratégico» aun cuando el modelo de partida fuera el «regionalismo abierto», lleva a plantear necesariamente algunas cuestiones conceptuales de base. Al definir el MERCOSUR como un instrumento estratégico, no solamente se hace referencia al modelo liberal que lo inspiró en su punto de partida, sino también a la respuesta política que articuló el Estado a partir de la percepción del gobierno, las elites y la burocracia principalmente, de las capacidades de participación en el escenario internacional y regional. Esta cuestión es fundamental para entender desde la perspectiva de Argentina y Brasil la apuesta al «regionalismo estratégico» en el siglo XXI, en la medida que existe una línea de continuidad en las prioridades de sus políticas exteriores, de mejorar la posición internacional, aun cuando hayan adquirido una importancia mayor los temas políticos y sociales, a partir de los cambios de gobierno.

La continuidad entre el regionalismo de los noventa del siglo pasado y el que se comenzó a esbozar a mediados de la primera década del siglo XXI en América del Sur, se produjo a partir de que algunos Estados, además de los países del MERCOSUR, visualizaron la respuesta regional como una herramienta de la política exterior para la inserción política internacional (y no solo competitiva). Es claro que al estar en función de la política exterior de los países, la resultante 
de los distintos procesos regionales dependía de los Estados componentes, de sus tradiciones políticas regionales y los recursos y capacidades concretos, por lo que básicamente quedaron plasmados dos modelos a mediados de los años noventa: el del Acuerdo de Libre Comercio de las Américas y el del MERCOSUR. De esta manera quedaron limitadas otras opciones de política exterior en las relaciones interamericanas y regionales de los países latinoamericanos (CERVO, 2001; MORGENFELD, 2009).

Uno de los puntos cruciales que surgen sobre el tema del «regionalismo estratégico» tiene relación con los fundamentos sobre los cuales se basa esa línea de continuidad, aun cuando se haya modificado el contexto internacional y también los temas y prioridades de la agenda regional y por ende la propuesta de base.

Esta pregunta, fue respondida desde la perspectiva de las relaciones internacionales, al enfatizar la continuidad de las políticas exteriores de algunos países latinoamericanos, que tuvieron como prioridad posicionarse mejor en el escenario internacional y pudieron hacer converger dicha prioridad con el «interés nacional» definido estratégicamente por algunos sectores económicos. Sin embargo, queda como interrogante si esa respuesta regional es funcional al capitalismo. En otras palabras, si la relación Estado-región-mundo tal cual se está procesando en el siglo XXI en algunos países de la periferia, que plantean un cambio del modelo neoliberal en el papel que debe jugar el Estado, es conducente con la evolución del capitalismo en su actual faceta global. El próximo apartado apunta a un esbozo de esclarecimiento de estos interrogantes y a plantear algunas consideraciones sobre la relación entre las respuestas regionales y el proceso de globalización.

\section{Estado y regiones en el sistema internacional del siglo XXI}

La caída del muro de Berlín aparejó no solamente el colapso de la Unión Soviética, sino también una modificación en las bases de funcionamiento del sistema internacional. Las repercusiones fueron importantes tanto en la profundidad de los cambios como en la horizontalidad de asuntos que en cascada ingresaron en la turbulencia del tiempo histórico.

El abrupto pasaje a una estructura unipolar en el sistema planteó dilemas de envergadura en Estados Unidos entre los analistas de las relaciones internacionales, aun cuando en los inicios se esbozó un «optimismo occidental del fin de la historia». El conjunto de situaciones históricas que se fueron procesando en distintos lugares del mundo (Balcanes, Europa Central, África), en los años noventa, erosionó totalmente esta perspectiva, planteando la necesidad de introducir nuevos elementos que posibilitaran respuestas diferentes y posibles a temas delicados de las relaciones internacionales (BADIE-SMOUTS, 1992; BADIE, 1995).

Las distintas respuestas analíticas post-fin de la historia reubicaron desde diferentes perspectivas algunos de los temas sensibles de la transición del sistema bipolar. Una de las perspectivas analíticas adoptadas, que comenzó a tener peso 
y a ser relevante en términos del funcionamiento del sistema internacional, fue la que consideró las consecuencias que aparejaba la mundialización del capitalismo en las lógicas de funcionamiento estatal, basadas en espacios «territoriales» definidos y en las respuestas de los Estados conformando nuevas instancias institucionales y espacios regionales.

La nueva organización del trabajo y la concomitante «desnacionalización» del producto aparejó varias consecuencias en el «espacio nacional conocido», en reconfigurar los territorios y ámbitos de decisión desde lo local y regional, y en el peso cada vez mayor que fueron adquiriendo otros articuladores del poder mundial (BECK, 2003; DUPAS, 2005; OHMAE, 1997).

Por otra parte, los procesos de regionalización que impulsaron desde inicios de los noventa la tríada Estados Unidos-Europa-Japón constituyeron respuestas específicas de inserción competitiva, que buscaron sentar bases para un mejor posicionamiento en el mercado mundial. Los países de la periferia no fueron parte de ese proceso de decisión de la regionalización, pero acompañaron el proceso de apertura y de inserción competitiva a través de distintas modalidades de regionalismo. Algunos países de América Latina fueron incorporados en las iniciativas de Estados Unidos, como fue el caso de México con la inclusión en el Tratado de Libre Comercio de América del Norte (TLCAN) (AIXLINE, 1996).

En esta primera etapa de nuevo regionalismo, las respuestas para los países de América del Sur, una vez que México pasó a formar parte del TLCAN, fueron básicamente de dos órdenes en la ecuación desarrollo/proyección internacional. Algunos países vieron la posibilidad de seguir el ejemplo mexicano para asegurar el acceso al mercado de Estados Unidos y para promover el desarrollo, a partir de una modernización en todos los indicadores vinculados a la competitividad. En esa dirección, la política exterior de esos países buscó generar condiciones para estar incluidos en las iniciativas de Estados Unidos. En otros países, el nivel y características del desarrollo alcanzado y el interés de sectores económicos que trasuntaron un «interés nacional» promovieron una respuesta de regionalismo que tuviera una perspectiva estratégica (HETTNE-INOTAI-SUNKEL, 2000).

Chile fue un ejemplo de «regionalismo abierto» que concretó un TLC con Estados Unidos y luego lo hizo con la Unión Europea y con otros países desarrollados. Por otra parte la conformación del MERCOSUR definió desde los inicios un regionalismo estratégico: si bien los países se posicionaron desde el Consenso de Washington y suscitaron una política de apertura económica, trazaron un límite en las negociaciones con la Unión Europea y con Estados Unidos (CARDOSO, 2000). Desde la perspectiva de Brasil, ese límite tuvo en cuenta el interés nacional-regional que partió de la base de las prioridades de desarrollo elaboradas por el sistema político-burocrático y las elites brasileñas. De esta manera, la elaboración de un "programa de desarrollo nacional» con anclas en las fronteras y en los países del entorno, posibilitó que Brasil contara con una herramienta de intercambio en la región y también para la región, que a su vez 
le sirvió para un mejor posicionamiento en el escenario internacional (COUTO, 2007; GALVÃO, 2009).

En sus inicios, el proceso de regionalización se presentó básicamente en su manifestación económica, de reformulación de la economía política y de inserción competitiva. En los últimos años, han tomado cada vez mayor presencia las manifestaciones vinculadas a los límites sistémicos de la globalización, que se expresan ecológicamente (alimentos, cambio climático, recursos naturales), energéticamente (recursos naturales, energías alternativas) y en la disponibilidad de recursos naturales (alimentos, energía, capacidades para continuar con el desarrollo). Los límites sistémicos se manifiestan en los distintos ámbitos internacionales y regionales y en los espacios de negociación.

De esta manera, la regionalización también adquiere un componente cada vez mayor de poder vinculado a los paquetes negociados en las nuevas instancias de negociación global. Como la lógica de negociación a nivel internacional continúa estando en manos del Estado, las capacidades de un Estado pasan a tener un valor agregado en la medida que puede «representar» una región y a su vez manejar recursos de poder en los distintos escenarios en que se negocian los temas energéticos, ecológicos y vinculados a los límites del desarrollo. De esta forma, la articulación Estado-región y de los contenidos e instrumentos que se otorgue al regionalismo pasa a constituir un elemento clave en la composición y definición de la estructura de poder internacional.

Estos diversos niveles en los que se expresa el regionalismo patenta la complejidad del fenómeno en cuanto una vía en que transitan permanentemente los flujos globales y las respuestas e iniciativas desde los Estados. Las capacidades de los Estados en aprovechar el regionalismo en cuanto articulador con el nivel mundial es un indicador de sus recursos y del incremento de su potencial, en particular de algunas de sus competencias. Por otra parte, las instituciones regionales se relacionan e insertan cada vez más con las estructuras económicas internacionales, lo que deriva en la construcción de un diseño-mundo que tiene distintos niveles (estatal, regional, mundial) y ámbitos (geopolítico, geoeconómico, comercial, tecnológico, sectorial) en las organizaciones multilaterales. En la medida que el regionalismo se expresa también en distintas instancias del escenario internacional, ya sea como bloque o como espacio diferenciado, tiene el potencial de generar ideas, iniciativas y participar en las instituciones y en la implementación de programas y acciones.

El regionalismo tiene en el siglo XXI, a diferencia del anterior, un punto de partida en la dimensión económica y política (internacional, regional, nacional y local), pero también posibilita viabilizar respuestas a los límites sistémicos de la globalización desde el Estado y hacia el mundo externo. Por otra parte, el regionalismo tiene una faceta social y de gobernanza en su presencia estructurante de un nuevo orden internacional y en su dimensión de articulación de los objetivos del milenio (ODM) y de otros objetivos incluyentes, como la cohesión social. 
En la década de los noventa la expresión de la gobernanza global por medio de los regionalismos se condensaba básicamente en el «regionalismo abierto» a través del modelo de Estados Unidos y de gobernanza «multinivel» europea. El modelo europeo buscó ser una propuesta diferenciada de la propuesta de regionalismo abierto, al modelo de Estados Unidos, por medio de la inclusión del diálogo político, la cooperación y la participación de la sociedad civil en las negociaciones de libre comercio con América Latina/Caribe y los procesos regionales comenzando por el MERCOSUR (BIZZOZERO, 2004; 2006; SANTANDER, 2004). El modelo asiático no contaba como propuesta en los noventa, porque mantenía una regulación importante del Estado y no daba entrada a la participación de la sociedad civil.

El cambio de contexto mundial en el siglo XXI provocó un retorno del papel del Estado y del debate sobre sus alcances, particularmente en la esfera de la seguridad. En América del Sur, la inclusión de la seguridad en la agenda formó parte de los temas del desarrollo regional. La crisis que se procesó en Argentina y otros países de la región a inicios del siglo, coadyuvó a que se plantearan de una manera diferente los contenidos del regionalismo en América del Sur. En particular, se fueron esbozando, a partir de los cambios de gobierno que se produjeron en los países de la región: una modificación de las prioridades, centrándolas en lo político y social; una vinculación del regionalismo con el debate estratégico sobre el orden internacional y su estructura; el planteo de iniciativas para desarrollar la región sudamericana como zona de paz y de cooperación, buscando además instrumentos para viabilizar su autonomía; y se comenzaron a visualizar otras posibles alternativas de desarrollo a partir de los recursos humanos y naturales «endógenos».

El retorno del Estado en tanto resguardo frente a las «amenazas externas», a lo cual ayudaron las crisis energética y de alimentos y posteriormente la crisis de aplicación del modelo neoliberal en varios países periféricos, antes de llegar a la financiero-económica, ha replanteado bajo nuevas perspectivas el tema de las cargas y responsabilidades de las potencias y su vinculación con el orden internacional. $\mathrm{El}$ «corto» siglo XXI ha traído varios debates a las relaciones internacionales cuyo eje es la transición del sistema y que tiene varios componentes como aristas. Uno de los puntos claves en cuanto a esta transición tiene que ver con el papel que pueden (o deban) jugar los denominados emergentes del sistema, las potencias medias y las regionales, entre las cuales se encuentra Brasil (GRATIUS, 2008). La cuestión radica entonces en visualizar la significación que puedan tener estas categorías y los contenidos que plantean en los ámbitos que se han gestado a partir de las potencias emergentes, ya sea en los objetivos, en las propuestas sobre el funcionamiento del sistema, o en los temas de la agenda.

El nuevo «nacionalismo estatal» no modifica sustancialmente las bases de funcionamiento del sistema ni la aceleración del proceso de mundialización del capitalismo. Lo que importa en la transición del nuevo orden, en la participación en la gobernanza global y también en la propuesta de desarrollo en cuanto 
manifestación de los recursos de poder, es la calidad del vínculo de los países denominados emergentes con el regionalismo y las capacidades para potenciar el regionalismo en el contexto global. En definitiva, el núcleo del debate en la transición del sistema internacional atañe a visualizar hacia qué estructura se encamina y al papel que cumplen las potencias emergentes (PEMS) en la manifestación regional y en la construcción del nuevo orden. A ello se agrega la específica manifestación del regionalismo como tal en la arquitectura global y nacional y en los distintos niveles de gobernanza.

El papel de las potencias emergentes en la construcción del nuevo orden no está desligado de la aceleración del proceso de mundialización del capitalismo, que a partir de los noventa incorporó países que estaban en el margen del sistema por consideraciones estratégicas frente a la competencia bipolar existente. El desempeño exitoso de las PEMS en el plano económico requiere necesariamente un entorno pacífico y desarrollado, ingredientes que deben ser encarados a través de instancias regionales.

El regionalismo representa para las potencias emergentes, entre las cuales se ubica Brasil, la contraparte política, social y cultural, además de mercados para el comercio. Por otra parte, el desempeño de las empresas a nivel regional precisa de un espacio en desarrollo, lo cual puede ser garantizado en buena medida por esos Estados-región que albergan (y sobre los cuales se asienta) el regionalismo. Como la política internacional de Estados Unidos durante la primera década del siglo XXI ha sido consecuentemente no regionalista (además de no multilateral), durante la presidencia de Bush, se ha manifestado como respuesta una mayor participación y representación de las potencias emergentes, medias y regionales, que atañe también a una perspectiva sobre las modalidades de gestión y regulación del capitalismo.

Una de las explicaciones secundarias para entender el interés actualizado en la participación de las PEMS en el orden internacional tiene que ver con los límites sistémicos globales y las repercusiones que los mismos generan. Esas repercusiones se han ido expresando en contradicciones en tres órdenes: en las relaciones entre Estados Unidos y el resto del mundo; al interior de los países desarrollados y finalmente en las relaciones centro-periferia.

De esta manera el regionalismo latinoamericano y sudamericano se ve confrontado a nuevos desafíos, además de los ya existentes, que atañen al papel de Estados Unidos en el espacio interamericano, la posibilidad de expresarse como región o conformarse con expresiones «fragmentadas» o incluso «nacionales»; y la de conformar un camino que vaya diseñando una respuesta estratégica en el sistema internacional.

Los nuevos desafíos en el espacio latinoamericano/caribeño atañen a la definición de respuestas frente a los límites sistémicos globales a los efectos de encontrar espacios de participación y negociación en los ámbitos internacionales/ regionales en que se encuentran representados. A su vez, la limitación de opciones 
regionales en términos de disyuntivas de relaciones internacionales y economía política acota las perspectivas de "salida» nacionales. Por ello, se van tejiendo otras redes y tejidos que dan lugar a nuevas modalidades de expresión de la cooperación y conflicto en las relaciones internacionales. La próxima sección se remitirá a esta diversidad de expresiones regionales en el espacio latinoamericano/caribeño, y a las opciones que se plantean frente al debate del regionalismo estratégico.

\section{El regionalismo latinoamericano del siglo XXI: niveles de participación en la gobernanza}

El sistema internacional de la posguerra fría, durante la década de los noventa, tuvo una estructura internacional de dos niveles: el estratégico-político, donde Estados Unidos actuó como el polo exclusivo, y el económico-político, donde se planteó una concertación multipolar con Estados Unidos como uno de los polos, además la Unión Europea y finalmente Japón. Sobre esta base se pretendió dar una respuesta a la estructuración del nuevo orden internacional, a través de una adecuación de las instituciones y del sistema de funcionamiento que pertenecía al orden de la bipolaridad.

Los primeros años del siglo XXI mostraron los intentos de Estados Unidos de gestionar los ajustes necesarios en los dos niveles para definir un orden internacional con determinados principios de funcionamiento, articular una nueva concertación internacional acoplada piramidalmente, realizar ajustes y plantear sobre nuevas bases los distintos ámbitos e instituciones internacionales existentes, y definir nuevos principios y reglas de funcionamiento en el derecho, la política y la economía internacional. El desgaste de las intervenciones en Afganistán e Irak aunado a la crisis económica, cuyo centro se inició en Estados Unidos con la crisis financiera de las hipotecas, el resquebrajamiento de la coalición de países y la falta de capacidades y recursos para encaminar una salida en distintas cumbres y negociaciones internacionales, plantearon las limitaciones del orden bosquejado a dos niveles bajo la órbita estadounidense.

La importancia asumida por las PEMS como consecuencia de su creciente participación en la economía mundial y en distintos ámbitos y foros de negociación y los límites sistémicos globales trajeron a su vez una nueva situación en el sistema internacional. La estructura del sistema ha tomado una nueva complejidad de varios niveles en que además del estratégico-político y el económico-político pueden encontrarse niveles atinentes a los límites sistémicos globales (energético, climático, alimentario). Si bien estos niveles están interrelacionados con los otros dos, al tomar esa relevancia sistémica pasan a tener una dimensión propia y específica. Esta nueva realidad estructural que pauta el funcionamiento del sistema internacional ha sido catalogada de distintas maneras. Una de ellas se refiere al mundo interpolar, porque el sistema estaría funcionado bajo un padrón de interdependencia multipolar (GREVI, 2009). 
Desde otro punto de vista, lo que importa es que los límites sistémicos y la participación de otros actores en la estructura dan lugar a una articulación en distintos niveles de la misma por parte de diferentes Estados y actores, algunos de los cuales se repiten, que pueden generar principios, normas y bases de políticas en los diferentes temas tratados. Esto hace que se deba analizar cada nivel y la articulación entre los distintos niveles para entender el funcionamiento actual del sistema internacional. Por esa razón resultan confusos los análisis que se centran en la caracterización del sistema, sobre la base de si el funcionamiento corresponde a un solo polo o a varios polos, porque el sistema es un resultado del conjunto de niveles y no posibilita analizar la complejidad del fenómeno en gestación.

Por otra parte, las PEMS han comenzado a participar en distintos espacios de negociación vinculados con temas diversos que tocan esos distintos niveles de la estructura internacional (GRATIUS, 2008). Los Estados emergentes además de presentarse en las esferas tradicionales como Estados, se presentan en instancias específicas como el BRIC (Brasil, Rusia, India, China) o el IBSA (India, Brasil, Sudáfrica) y conforman otros grupos y redes ya sea en las regiones o con diversos Estados en las negociaciones internacionales como han sido los casos de los grupos generados en el marco de la Ronda de Doha para el Desarrollo (SOUZA, 2008).

Las regiones pueden potenciarse específicamente en alguno de los niveles y/o proyectar solamente la posición del Estado-región. En cualquiera de los casos lo que importa es la vinculación entre el nivel regional y el internacional, si el mismo esboza una función de adaptación a los requerimientos internacionales o de participación activa en las definiciones internacionales del nivel analizado.

Los niveles de la estructura internacional atañen en primer lugar al funcionamiento del sistema y a los pilares básicos sobre los cuales "funciona» tanto en términos estratégicos-políticos como económicos, es decir lo tradicional atinente al poder y la jerarquía en el sistema. En segundo orden los niveles conciernen a los recursos institucionales, económicos, tecnológicos y científicos. En tercer lugar, se han conformado niveles en el sistema a partir de los límites sistémicos globales (energía, medio ambiente, cambio climático, recursos alimentarios, demografía). En los niveles del segundo y tercer orden se plantean además de las tradicionales negociaciones de poder que tienen como base el primer nivel, modelos de gobernanza global. Como los regionalismos traen consigo un modelo de gobernanza regional, la participación de los Estados referentes y mismo de los bloques regionales puede aportar elementos para conformar un espacio de negociación (y de poder).

Los procesos de cooperación e integración regional de América Latina y Caribe expresan en el presente las diferentes lógicas e historias en los cuales se han gestado y han ido evolucionando. A su vez los espacios interregionales, continentales y eurolatinoamericanos/caribeños reflejan en ese nivel circunscrito, modelos de gobernanza diferentes en distintos niveles, lo que se traduce en propuestas políticas y de cooperación de distinto alcance. 
De esta manera, América Latina (y también el Caribe, en la medida que recientemente se creó un espacio específico a través de la Cumbre América Latina/ Caribe), expresan una diversidad de procesos regionales y de espacios interregionales, continentales, eurolatinoamericanos, además de otros donde participan algunos Estados como los países del Pacífico y otros ad hoc que se van creando en diversas cumbres. Sin embargo, la cuestión esencial se remite a si esos procesos regionales expresan un regionalismo estratégico o bien si los procesos regionales básicamente tienen como función adaptar y aplicar las políticas concertadas en instancias internacionales como es el caso de los Objetivos del Milenio.

En ese sentido, América Latina ha heredado en el siglo XXI, las opciones y los condicionantes estructurales de la década de los noventa, que se traducen en distintas orientaciones de política exterior atinentes a la posición en la estructura del sistema internacional por parte de los Estados y diferentes concepciones de desarrollo. Como en definitiva, el «interés nacional» está en función de la posición del país en la región y el mundo, lo que importa para definir si un proceso regional es (o intenta ser) estratégico, tiene que ver con las definiciones de política exterior. Por ello, el escalonamiento MERCOSUR/UNASUR resulta para Brasil (y en otra medida para Argentina) estratégico en el plano mundial. A su vez la conformación del espacio América Latina/Caribe tiene que ver con las prioridades de México para no quedar aislado de los procesos regionales y para mantener márgenes de autonomía que le posibiliten generar espacios de participación en los niveles considerados de la estructura internacional.

En definitiva, la coyuntura internacional ha facilitado la conformación de nuevas instancias regionales en América Latina y el Caribe que trasuntan modelos específicos de gobernanza y que expresan las prioridades de las políticas exteriores de los principales actores de la región. Por otra parte, se ha seguido consolidando un espacio sudamericano, el cual todavía tiene por delante varios desafíos atinentes al modelo de gobernanza, la participación de los Estados, de las regiones, las sociedades y sobre todo la inclusión de las diferentes comunidades nativas.

Sin embargo, a pesar de que varias señales apuntan a que el espacio sudamericano pueda ser un polo en los distintos niveles de la estructura del sistema internacional o en alguno de ellos e incluso posibilitar la ampliación a un espacio latinoamericano/caribeño, se plantean varios desafíos y escollos. Entre esos desafíos, algunos provienen de las clásicas políticas estadounidenses, pero habrá en el futuro otros provenientes de otros actores de fuera de la región, como es el caso de China. Otros desafíos provendrán de las diferencias entre las políticas exteriores de los países de la región en la construcción regional y de la expresión de la política exterior brasileña en la región (LIMA, 2005; SOUTO MAIOR, 2006; BERNAL-MEZA, 2008). Finalmente, un aspecto de fundamental consideración atañe a la propia construcción regional: sus contenidos, la capacidad de los Estados de generar instancias de participación de las regiones, comunidades nativas y 
actores de la sociedad civil y en procesar las políticas necesarias para generar bienes públicos regionales.

\section{Conclusiones}

Los procesos regionales que se iniciaron en los noventa en América Latina partieron del común denominador del «Consenso de Washington», que definió como uno de los objetivos la inserción competitiva. Si bien los Estados en los procesos regionales se guiaron por estos parámetros, las políticas exteriores de Argentina y sobre todo de Brasil ubicaron al MERCOSUR como una prioridad para potenciar sus posibilidades en el escenario internacional. De esta manera, el MERCOSUR fue desde los años noventa un regionalismo estratégico, lo cual posibilitó una continuidad con los cambios que se produjeron durante el siglo XXI.

Una de las cuestiones que se plantean para visualizar la relevancia de los regionalismos en el siglo XXI es la constatación de la importancia que han tomado las potencias emergentes en el sistema internacional. Como la fase actual del capitalismo ha planteado el surgimiento de varias potencias emergentes que están tomando un mayor protagonismo internacional, se refleja en la estructura del sistema internacional y en la necesidad de nuevas regulaciones. Por ello, las regiones y los procesos regionales toman importancia en el funcionamiento del sistema internacional y en su orientación, en la medida que se plantean distintos modelos de gobernanza.

Por otra parte, se están manifestando límites sistémicos en el sistema internacional que generan consecuencias específicas en las potencias emergentes, los Estados y las regiones. Estos límites están planteando de otra forma la ecuación del poder y la relación entre los Estados y el sistema internacional. En esta nueva configuración, las regiones y el Estado-región tienen otro papel en el sistema internacional.

El escenario que se perfila para la segunda década del siglo XXI presenta un abanico de opciones estratégicas para los Estados de la región latinoamericana/ caribeña, que podrán potenciarlas en las instancias regionales, para negociar estratégicamente o bien para conformar negociaciones puntuales. Habrá dos ejes que serán relevantes en todo el proceso durante los próximos años: el que atañe a la participación del Estado en la estructura del sistema internacional en alguno de los niveles y el que se vincula con la construcción del modelo de gobernanza regional. En el primer eje importa específicamente la articulación del Estado con la región (Estado-región) y su capacidad de competir y asociarse con otros Estados. En el segundo eje, resultan relevantes los mecanismos institucionales y políticos que se van generando para propiciar bases de una sociedad regional, inclusiva y ambientalmente sustentable. 


\section{Bibliografía}

AIXLINE, Andrew: «Regionalismo latinoamericano en la era de la globalización» en BIZZOZERO, Lincoln y VAILLANT, Marcel: La inserción internacional del MERCOSUR. ¿Mirando al Sur o mirando al Norte? Montevideo: ARCA, 1996, p. 77-98.

BADIE, Bertrand y SMOUTS, Claude: Le retournement du monde. Sociologie de la scène politique internationale, Paris: Dalloz, 1992.

BADIE, Bertrand: La fin des territoires. Essai sur le désordre international et sur l'utilité sociale du respect, Paris: Fayard, 1995.

BECK, Ulrich: Pouvoir et contre-pouvoir à l'ère de la mondialisation, Paris: Flammarion, 2003.

BERNAL-MEZA, Raúl: Argentina y Brasil en la Política Internacional: regionalismo y Mercosur (estrategias, cooperación y factores de tensión) Revista Brasileira de Política Internacional 2008, vol. 51, n. 2, p. 154-178.

BIZZOZERO, Lincoln: Las relaciones MERCOSUR-Unión Europea. Una nueva modalidad de participación de las regiones en la mundialización, en GÓMEZ, José María (comp.): América Latina y el (des)orden global neoliberal. Hegemonía, contrahegemonía y perspectivas, Buenos Aires: CLACSO, 2004, p. 97-116.

BIZZOZERO, Lincoln: Negociaciones MERCOSUR-Unión Europea, articulación del espacio Euro-Latinoamericano/Caribeño y gobernanza mundial, Cuadernos de Integración Europea n. ${ }^{\circ}$ 5, Centre de Documentació Europea de la Fundació General de la Universitat de València, 2006, p. 5-27.

CARDOSO, Fernando Henrique: Discurso do Presidente da República na Solenidade de Formatura dos Alunos do Instituto Rio Branco, Palácio Itamaraty, 18/05/2000. Publicado en: <http://ftp.unb.br/pub/UnB/ipr/rel/discpr/2000/2916.pdf> [Consulta: 21 ene. 2010].

CERVO, Amado Luiz: Relaçôes internacionais da América Latina. Velhos e novos paradigmas, Brasília: IBRI-FUNAG, 2001.

COUTO, Leandro: O horizonte regional do Brasil e a construção da América do Sul, Revista Brasileira de Politica Internacional, vol. 50, n. ${ }^{\circ}$ 1, 2007, p. 159-176.

DUPAS, Gilberto: Nova Ordem Global e a Política. O espaço da periferia, Publicado en Red de Bibliotecas Virtuales de Ciencias Sociales de América Latina y el Caribe de la Red de Centros Miembros de CLACSO, 2005, <http://bibliotecavirtual.clacso.org.ar/ar/libros/reggen/pp15. pdf> [Consulta: 5 nov. 2009].

GALVÀO, Thiago Gehre: América do Sul: construção pela reinvenção (2000-2008) Revista Brasileira de Politica Internacional, Vol. 52, n. ${ }^{\circ} 2,2009$, p. 63-80.

GRATIUS, Susane: Las potencias emergentes: ¡estabilizadoras o desestabilizadoras?, Publicado en Comentario n. ${ }^{\circ}$ 3, Madrid, Fundación para las Relaciones Internacionales y el Diálogo Exterior. <http://www.fride.org/publicacion/414/las-potencias-emergentes-estabilizadoraso-desestabilizadoras> [Consulta: 18 ene. 2010].

GREVI, Giovanni: The interpolar world: a new scenario, Institute for Security Studies Occasional Papers, n. ${ }^{\circ 79}$, junio 2009, 44 p. Publicado en The Institute for Security Studies, 18 ene. 2010.

HETTNE, Björn, INOTAI, András y SUNKEL, Osvaldo: National Perspectives on the New Regionalism in the South Helsinki, Londres: St. Martin's Press, Mac. Millan Press, 2000.

LIMA, Maria Regina Soares de: A política externa brasileira e os desafios da cooperação Sul-Sul, Revista Brasileira de Politica Internacional, vol. 48, n. ${ }^{\circ}$ 1, 2005, p. $24-59$. 
MORGENFELD, Leandro Ariel: Un antecedente histórico del fracaso del ALCA: la Argentina frente a la unión aduanera americana propuesta en Washington en 1889, Realidad Económica, n. ${ }^{\circ}$ 246, Buenos Aires, 2009, Publicado por IADE: <http://www.iade.org.ar/modules/ RealidadEconomica/index.php?categoria $=5106>$ [Consulta: 12 dic. 2009].

OHMAE, Kenichi: El fin del Estado-Nación. El ascenso de las economías regionales, Barcelona, Buenos Aires, México, Santiago de Chile: Editorial Andrés Bello, 1997.

SANTANDER, Sebastián: Globalisastion, gouvernance et logiques régionales dans les Amériques en SANTANDER, Sebastián (coord.): Globalisastion, gouvernance et logiques régionales dans les Amériques Cahiers du GELA-IS. Bruselas: L'Harmattan, GELA-IS, p. 5-42.

SOUTO MAIOR, Luiz A. P.: O Brasil e o regionalismo continental frente a uma ordem mundial em transição. Revista Brasileira de Política Internacional, Vol. 49, n. ${ }^{\circ}$ 2, 2006, p. $42-59$.

SOUZA, Sarah-Lea John de: Brasil, India y Sudáfrica, potencias para un nuevo orden, Política Exterior, n. ${ }^{\circ}$ 121, enero-febrero, p. 165-178, 2008, Publicado por FRIDE, <http://www.fride. org/publicacion/346/brasil-india-y-sudafrica-potencias-para-un-nuevo-orden> [Consulta: 12 dic. 2009].

VIGEVANI, Tullo y RAMANZINI Jr., Haroldo: Brasil en el centro de la integración Nueva Sociedad n. ${ }^{\circ}$ 219, Caracas, enero-febrero 2009, p. 76-96.

Recibido en 3 de marzo de 2010 Aceptado el 8 de noviembre de 2010

\section{Resumen}

El artículo analiza la evolución del regionalismo en América Latina desde los años noventa. Expone las opciones que se plantean a los países en sus políticas exteriores frente a las iniciativas de Estados Unidos y los cambios en el sistema internacional. Se expone la importancia funcional de las potencias emergentes (PEMS) y las regiones en la fase actual del capitalismo. Finalmente el artículo analiza las perspectivas del regionalismo en América Latina tanto en el sistema internacional como en la construcción de un modelo de gobernanza regional.

Palabras clave: América Latina; regionalismo estratégico; potencias emergentes; gobernanza regional; gobernanza global.

\section{Abstract}

The article analyzes the evolution of regionalism in Latin America from the 1990s. It exhibits the options that consider to the countries in their foreign policies against the initiatives of the United States and the changes in the international system. One sets out the functional importance of the emergent powers (PEMS) and the regions in the present phase of Capitalism. Finally, the article analyzes the perspective of regionalism in Latin America as much in the international system as in the construction of a regional model of governance.

Keywords: Latin America; strategic regionalism; emerging powers; regional governance; global governance. 